\title{
A Novel Approach for Salt and Pepper Noise Removal using ROR and Contourlet Transform
}

\author{
M. Mohana Dhas \\ Associative Professor\&Head \\ Department of M.C.A., \\ Annai Velankanni College, \\ Tholayavattam, Tamilnadu, India
}

\begin{abstract}
ROR and Contourlet transform is proposed to restore images corrupted by salt and pepper impulse noise. The operation is carried out in two stages, i.e., detection followed by filtering. For detection first, we propose the robust outlyingness ratio (ROR) for measuring how impulse like each pixel is, and then all the pixels are divided into four clusters according to the ROR values. Second, different decision rules are used to detect the impulse noise based on the absolute deviation to the median in each cluster. In order to make the detection results more accurate and more robust, the from-coarse-to-fine strategy and the iterative framework are used. In addition, the detection procedure consists of two stages, i.e., the coarse and fine detection stages. For filtering, proposed algorithm using contourlet transform (CT). Simulation results demonstrate that the proposed algorithm is better than traditional filters and is particularly effective for the cases where the images are very highly corrupted.
\end{abstract}

Keywords - ROR, impulse noise, Contourlet transform, denoising.

\section{INTRODUCTION}

DIGITAL images are often corrupted by impulse noise due to transmission errors, malfunctioning pixel elements in the camera sensors, faulty memory locations, and timing errors in analog-to-digital conversion[1]. Here some improved algorithms are proposed to eliminate salt and pepper noise. In most applications, denoising the image is fundamental to subsequent image processing operations, such as edge detection, image segmentation, object recognition, etc. The goal of noise removal is to suppress the noise while preserving image details. To this end, a variety of techniques have been proposed to remove impulse noise.

Several methods have been proposed to remove the noise and recover the true image. The corrupted pixels should be filtered, whereas the image details have to be preserved. This task is exceptionally difficult because even the smallest amount of noise impulses which are not detected and filtered cause significant deterioration of image quality due to the nature of impulse noise. There have been proposed a large number of filtering techniques for removal of impulse noise.

Switching median (SM) filter [2], multistate median (MSM) filter [3], adaptive center weighted median (ACWM) filter [4], the peak-and-valley filter [5], [6], signal-dependent rank-order mean (SD-ROM) filter [7], conditional signaladaptive median (CSAM) filter [8], the pixel-wise MAD (PWMAD) filter [9], modified threshold Boolean filter (TBF) [10], Jarque-Bera test based median (JM) filter [11], twooutput nonlinear filter [12], iterative median filter [13], etc. With the noise detector, these filters will check each pixel if it is corrupted or not. Then, only noisy pixels are changed so as to avoid undue distortion. The main drawback of these filters is that they just use median values or their variations to restore

\author{
G.Suganthi,Ph.D \\ Associative Professor \\ Department of computer science, Women \\ Christian College,Nagercoil, Tamilnadu, India
}

the noisy pixels, and hence they usually cannot preserve the image details even when the images are mildly corrupted. Recently, Do and Vetterli [14], [15] developed the contourlet transform based on an efficient two-dimensional multi scale and directional filter bank that can deal effectively with images having smooth contours. Contourlets not only possess the main features of wavelets (namely, multi scale and timefrequency localization), but also offer a high degree of directionality and anisotropy. The main difference between contourlet and other multi scale directional systems is that the contourlet transform allows for different and flexible number of directions at each scale, while achieving nearly critical sampling. In addition, the contourlet transform uses iterated filter banks, which makes it computationally efficient; specifically, it requires $\mathrm{O}(\mathrm{N})$ operations for an $\mathrm{N}$-pixel image. In this paper, we propose a noise detector called robust outlyingness ratio (ROR), which is used to measure how much a pixel looks like an impulse noise[16]. Based on the ROR, the pixels are divided into four different clusters. Then, different decision rules are adopted to detect the impulse noise in each cluster. During the detection process, the from coarseto-fine strategy is used. In addition, the detection process contains two stages, i.e., the coarse stage followed by the fine stage. Different thresholds are used in the two stages. Finally, the detection procedure is iteratively adopted. Furthermore, Contourlet transform is a multidirectional and multi scale transform that is constructed by combining the Laplacian pyramid with directional filter bank (DFB), can be used to capture geometrical properties of images. After contourlet decomposition feature like edges have higher contourlet coefficient in high frequency band however noise having small contourlet transformation coefficient. Therefore, eliminating noise is to eliminating the smaller coefficients.

The rest of this paper is organized as follows. The ROR noise detection mechanisms are introduced in Section II. The extension with Contourlet transform for salt and pepper noise is described in Section III. Section IV gives an Overall Discussion of the proposed algorithm. Finally, a brief Conclusion of this paper is presented in Section V.

\section{ROR FOR SALT AND PEPPER NOISE DETECTION}

\section{A. Noise Models}

When an image is corrupted by impulse noise, only a portion of the pixels are changed. To be precise, let $x_{i j}$ and $\eta_{0}$ be the pixel values at location in the original and noisy images respectively, $\left[n_{\text {min }} n_{\text {max }}\right]$ and be the dynamic range of the allowed pixel values. Then, for an impulse model with noise probability or noise ratio $\mathrm{P}$, we have

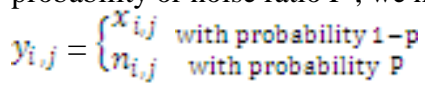

where $n_{L_{0} j}$ is a noise value independent from $x_{i, j}$. For fixedvalued impulse noise, the values of the corrupted pixels are 
equal to $n_{\text {ming }}$ or $n_{\text {max }}$ with equal probability [17]. For graylevel images with 8 bits per pixel (i.e., $n_{\text {min }}=0$ and $n_{\mathrm{max}}=255$ ), the noise value $n_{\mathrm{i} j \mathrm{j}}$ of the first case corresponds to a fixed value of 0 or 255 with equal probability.

\section{B. Definition of the ROR}

In this paper, we introduce it to describe the level of how impulse like of each pixel. The traditional sample mean and SD are used. The normalized median absolute deviation $(\mathrm{MADN})$ is defined as

$$
\begin{aligned}
& \operatorname{MADN}(y)=\operatorname{MAD}(y) / 0.6457 \\
& \operatorname{MAD}(y)=\operatorname{Med}\{\mid y-\operatorname{Med}(y)\} \\
& \operatorname{Med}(y)=\operatorname{Median}(y 1, y 2, \ldots y n)
\end{aligned}
$$

where the operation "Median" represents the median value of all the observations, the coefficient " 0.6457 " is the median absolute deviation (MAD) of a standard normal random variable, and $\mathrm{y}$ is the vector representation of the data. We define a statistical parameter called ROR as

\section{$\operatorname{ROR}\left(\mathrm{y}_{\mathrm{ij}}\right)=\left\|\left(\mathrm{y}_{\mathrm{i} j}-\operatorname{Med}(\mathrm{y})\right)\right\|_{/ \mathrm{MADN}}(\mathrm{y})(5)$ \\ C. Detection Mechanism}

In order to calculate the ROR, the window size must be given. In this paper, we use the window size is $5 X 5$. Since the ROR measures the outlyingness of the pixels, i.e., how impulse like, all pixels are divided into four levels (clusters) according to the ROR. The four clusters are the most like cluster $\mathrm{ROR}>3$; the second like cluster $2<R O R \leq 3$; the third like cluster $1<R O R \leq 2$; and the fourth like cluster $0 \leq R O R \leq 1$; The lower the ROR, the lower impulse like of the pixel in its neighbors.

\section{The coarse stage.}

Step 1) Choose the algorithm parameters, i.e., coarse thresholds $T_{1}^{E}, T_{2}^{E}$, and $T_{2}^{E}$; window size $\mathrm{N}$ (the actual size is $(2 N+1) \times(2 N+1)$; iterations $m_{e} ;$ and initial $\tilde{\eta}=1$.

Step 2) Initialize the detection flag matrix Map as zeros, where " $0 \mathrm{~s}$ " and " $1 \mathrm{~s}$ " represent good and noisy pixels, respectively.

Step 3) Calculate the ROR of the current pixel. If the ROR is in the fourth level, treat it as a good pixel, or calculate the absolute deviation $\mathrm{d}$ between the current pixel and the median of its local window. Then, compare with threshold $T_{\mathbb{E}}^{E}$ according to its ROR value. If $\mathrm{d}$ is larger than $T_{\mathrm{E}}^{\mathrm{E}}$, it is a noisy pixel, or it is a good pixel. Update the flag Map according to the result.

Step 4) Get the median-based restored image according to the detection result. If the flag is 1 , represent the pixel with the median of its local window, or do not change.

Step 5) If $j \leq m_{\varepsilon}, j=j+1$, then go to step 2), or the coarse stage is completed.

\section{The fine stage:}

Step 1) Choose the algorithm parameters, i.e., fine thresholds $T_{1}^{f}, T_{2}^{f}, T_{a}^{f}$ and $T_{4}^{f}$; window size $\mathrm{N}$ (the actual size is $(2 N+1) \times(2 N+1)$ ); iterations $m_{f}$; and initial $f=1$.

Step 2) Initialize the detection flag matrix Map as zeros, where " $0 \mathrm{~s}$ " and " $1 \mathrm{~s}$ " represent good and noisy pixels, respectively.

Step 3) Calculate the ROR of the current pixel and the absolute deviation between the current pixel and the median of its local window. Then, compare $d$ with threshold $T_{\mathrm{k}}^{f}$ according to its ROR value. If $\mathrm{d}$ is larger than $T_{\mathrm{f}}^{f}$, it is a noisy pixel, or it is a good pixel. Update the flag Map according to the result.

Step 4) Get the median-based restored image with the detection result. If the flag is 1 , represent the pixel with the median of its local window, or do not change.

Step 5) If $j \leq m_{f}, j=j+1$, , then go to step 2), or the fine stage is completed.

It is worth noting that the first input image of the fine stage is the final output image of the coarse stage. In the new detection mechanism, there are many parameters needing to be pre given, such as window size $\mathrm{N}$; coarse thresholds $T_{1}^{e} T_{2}^{e}$ and $T_{g}^{E}$; coarse iterations $m_{e}$; fine thresholds $T_{1}^{f} T_{2}^{f} T_{0} T_{a}^{f}$ and $T_{4}^{f}$; and fine iterations $m_{e}$. Theoretically, the thresholds dominate the performance of the detection algorithm. If the larger thresholds are used, the "false-hit" term is lower and the "miss" term is higher, whereas if the smaller thresholds are used, the results are opposite. In the coarse stage, the purpose is to detect the most impulse like pixels and keep the "false-hit" term as low as possible, and then in the fine stage, the purpose is to detect the most impulse like noise while keeping a good tradeoff between the "false-hit" and "miss" terms. Therefore, the thresholds of the coarse stage are relatively larger, and the thresholds of the fine stage are smaller. Furthermore, the larger the ROR values, the more impulse like the pixels; hence, the smaller thresholds should be used to guarantee detecting most of the noisy pixels. In addition, the smaller ROR values represent that the pixels are not like the impulse noise; hence, the larger thresholds should be adopted to guarantee detecting the most like impulse noise while making the "false-hit" term as small as possible.

In the four levels, the thresholds meet the following inequalities: $T_{1}^{E} \leq T_{2}^{E} \leq T_{\mathbb{q}}^{E}$ and $T_{1}^{f} \leq T_{2}^{f} \leq T_{\mathbb{q}}^{f} \leq T_{4}^{f}$. Fortunately, these parameters are very robust to the noise ratio, i.e., for different noise ratios, the thresholds and the window size can take the same values; only the iterative times are different. Through extensive experiments, the following suitable parameters are obtained: for random valued impulse noise, the window size is $\mathbb{N}=2$, i.e., $5 \times 5$; the coarse threshold is [30, 40, 50] for $T_{1}^{E}, T_{2}^{E}$ and $T_{a}^{E}$, respectively; the fine threshold is $[7, \quad 10,20,25]$ for $T_{1}^{f}, T_{2}^{f}, T_{\mathbb{a}}^{f}$ and $T_{4}^{f}$ respectively; for the fixed-valued impulse noise, the window size is $N=2$, i.e., $5 \times 5$; the coarse threshold is $[40,50,60]$ for $T_{1}^{E}, T_{2}^{E}$ and $T_{\mathbb{g}}^{E}$ respectively; the fine threshold is $[30,30,40,50]$ for $T_{1}^{f}, T_{2}^{f}, T_{\mathbb{a}}^{f}$ and $T_{4}^{f}$, respectively. 


\section{CONTOURLET NOISE REDUCTION}

\section{A. Contourlet Transformation}

The contourlet transform consists of two major stages: the sub band decomposition and the directional transform. At the first stage, Laplace Pyramid (LP) is used to decompose the image into sub band, and then the second one is a Directional Filter Bank (DFB) which is used to analyze each detail image. A flow graph of the CT is shown in Fig. 2. The contourlet transform was proposed as a directional multi resolution image representation that can efficiently capture and represent singularities along smooth object boundaries in natural images. Its efficient filter bank construction as well as low redundancy make it an attractive computational framework for various image processing applications. However, a major drawback of the original contourlet construction is that its basis images are not localized in the frequency domain [18]. Figure 1: A flow graph of the contourlet transform. Image is first decomposed into subbands by LP and then each detail image is analyzed by DFB.

.LP .DFB

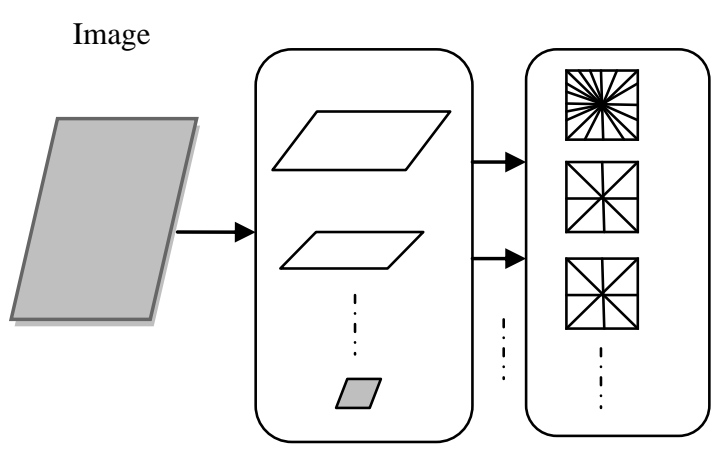

\section{B. Laplace pyramid}

The Laplace filters in the transform toolbar for images detect rapid changes in pixels using two different analytic matrices. The Laplace filters often result in "negative" images with brighter tones at detected features and dark or black tones elsewhere. These filters will emphasize linear features such as edges. In the first stage of the decomposition, the original image is transformed into a coarse signal and a detail signal. The coarse signal has fewer samples than the original image but the detail signal has the same number of samples as the original image. The coarse signal is a filtered and down sampled version of the original image. It is then up sampled and filtered to predict the original image. The prediction residual constitutes the detail signal. The coarse signal can be decomposed, further and this process can be repeated a few times iteratively. This yields a pyramid consisting of the coarsest version thus obtained and the detail signals at various scales. This pyramid is called Laplace pyramid [19].

\section{The directional filter banks}

Directional Filter Bank (DFB) is designed to capture the high frequency (representing directionality) of the processing image. Bamberger and Smith constructed a two-dimensional (2-D) DFB that can be maximally decimated while achieving perfect reconstruction. A new construction for the DFB to avoid modulating input image is proposed, by which we can obtain the desired 2-D spectrum division as shown in Fig. 2(a). The simplified DFB is intuitively constructed from two building blocks. The first is a 2-D spectrum into two directions: horizontal and vertical, as shown in Fig. 2(b). The second is a shearing operator, which is used to reordering the image samples. By appropriate combination of shearing operators together with two-direction partition of quincunx filter banks at each node in a binary tree-structured filter bank, shown in Fig.2(c), the CT structure is obtained.

Figure 2: The directional filter bank.

(a) Frequency partitioning.
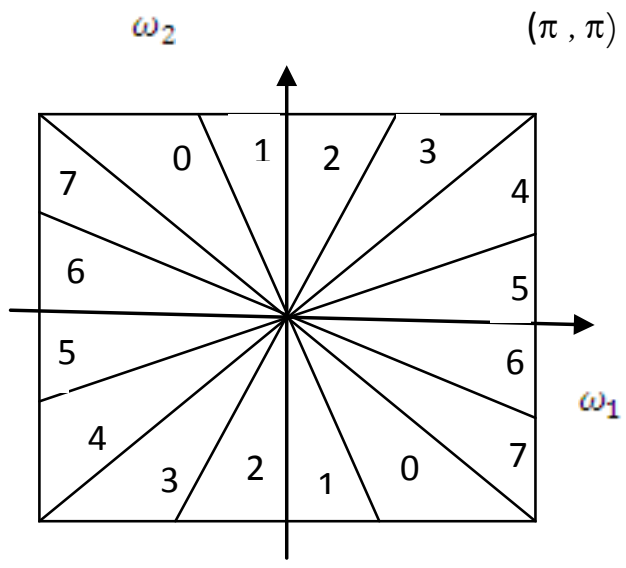

$(-\pi,-\pi)$

(b) 2-D spectrum partition using quincunx filter banks with fan filters.

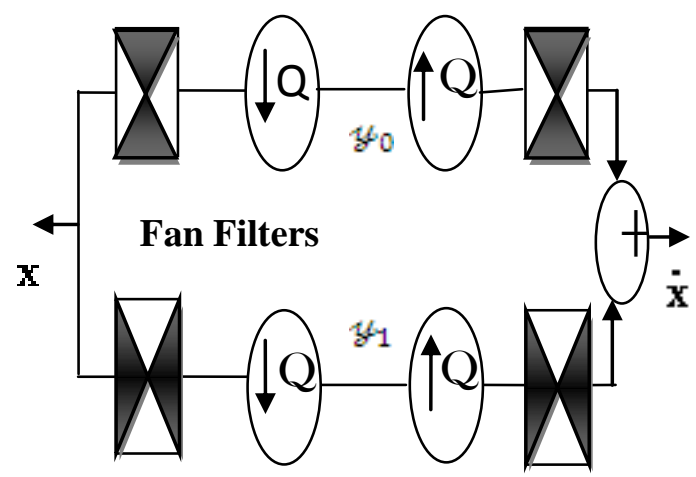

(c) The multichannel view of a one level tree structured DFB, Ei's, (i= 0,1,2,.. 2i-1), are the analysis filter functions, Di's, $(\mathrm{i}=0,1,2, . .2 \mathrm{i}-1)$, are the synthesis filter functions, and $\mathrm{Si}$ 's, (i= $0,1,2, \ldots 2 \mathrm{i}-1)$, are either the downer or up- samplers.

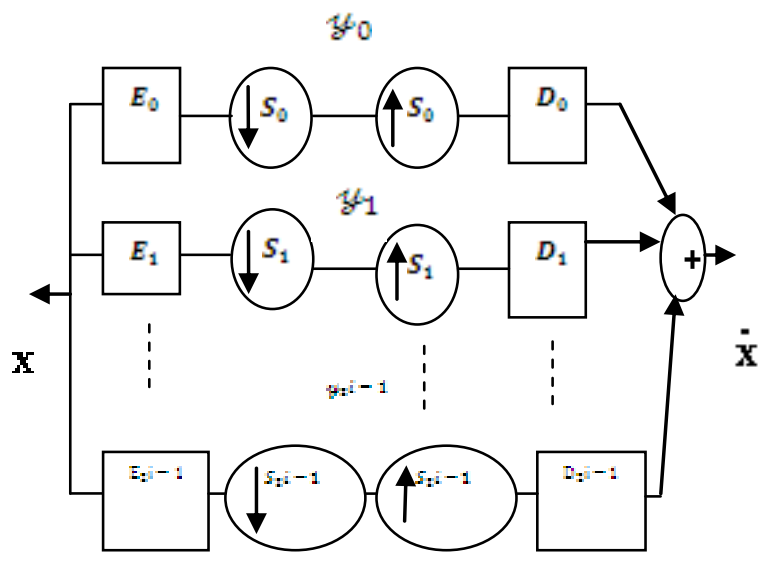




\section{EXPERIMENTAL RESULTS AND DISCUSSIONS}

The performance of the proposed ROR and Contourlet transform is tested against the ROR with nonlocal means (NLmeans) on a various noise ratio and PSNR value which are used for analysis listed here.

Figure 3 : Graph of PSNR and MSE values of (a) ROR with NL means and (b) ROR and Contourlet transform.

(a)

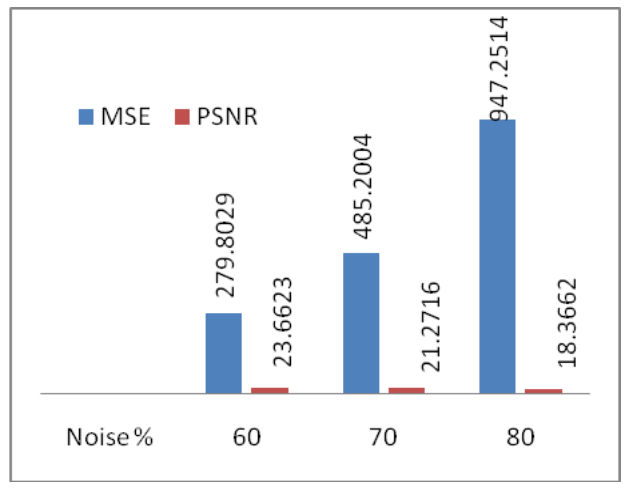

(b)

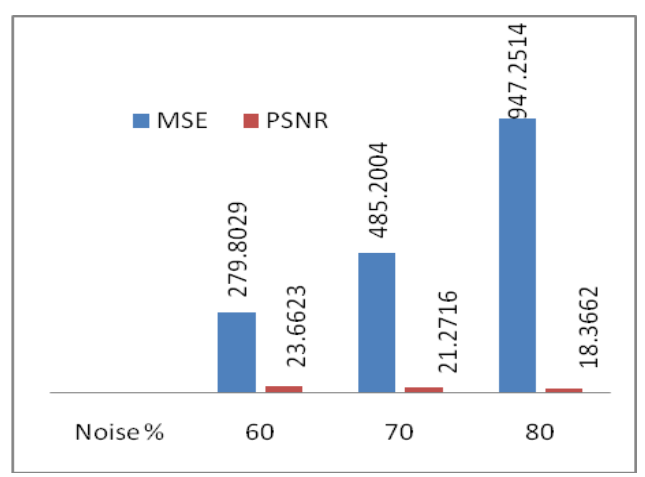

Objective comparisons of the performances of these filters (ROR with NL means, ROR and Contourlet transform) images corrupted by various levels of impulse noise ratios are made with the Mean Absolute Error (MAE) values and the Peak Signal to Noise ratios (PSNR) of the images restored by them.

PSNR $=10 \log _{10} \frac{(\mathrm{R})^{2}}{\mathrm{MSE}}$

where Mean Square Error, (MSE) is

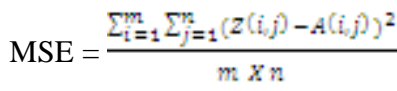

$\mathrm{R}$ - Maximum fluctuation. By default 255.

$\mathrm{Z}$ - Original Image.

A - Restore image.

$\mathrm{m}$ - number of rows.

$\mathrm{n}$ - number of columns.

Figure 4: (a) "Lena" image corrupted with $60 \%, 70 \%$ and $80 \%$ of salt-and-pepper noise. Results obtained using : (b) ROR with NL means (c) ROR and Contourlet transform. (a)

$60 \%$ Noise $\quad 70 \%$ Noise $\quad 80 \%$ Noise
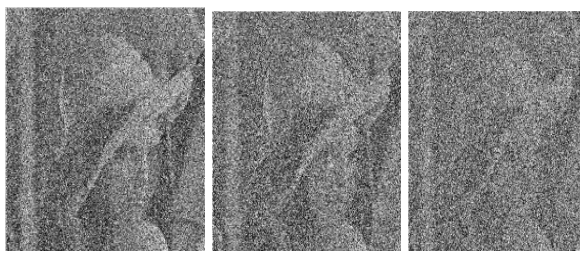

(b)

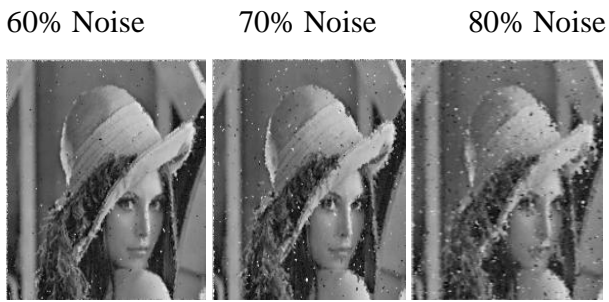

(c)

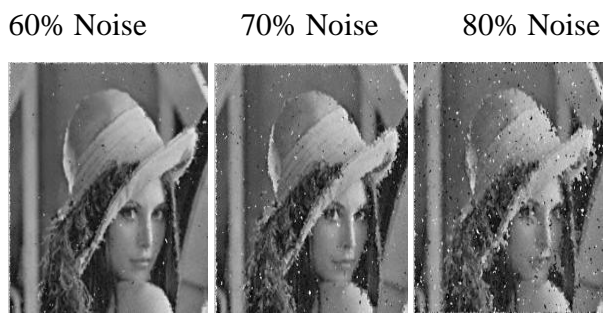

Table : Comparative Results of PSNR, MSE at different noise Densities for LENA image of (a) ROR with NL means (b) ROR with Contourlet transform.

(a)

\begin{tabular}{|c|c|c|}
\hline Noise $\%$ & MSE & PSNR \\
\hline 60 & 279.8029 & 23.6623 \\
\hline 70 & 485.2004 & 21.2716 \\
\hline 80 & 947.2514 & 18.3662 \\
\hline
\end{tabular}

(b)

\begin{tabular}{|c|c|c|}
\hline Noise $\%$ & MSE & PSNR \\
\hline 60 & 234.5778 & 24.4279 \\
\hline 70 & 406.3953 & 22.0413 \\
\hline 80 & 820.1520 & 18.9919 \\
\hline
\end{tabular}

\section{CONCLUSION}

In this paper, the ROR and Contourlet transform for identify more noisy pixels with less false hits and removal of salt andpepper noise is presented. We combine it with ROR and Contourlet to get a powerful method for removing salt and pepper noise. Simulation results show that our method outperforms with existing method both visually and quantitatively. In applications such as image denoising, we show the proposed new Contourlet construction significantly outperforms the original transform. 


\section{REFERENCES}

[1] R.C. Gonzalez and R.E. Woods, "Digital Image Processing," Englewood Cliffs, NJ: Prentice-Hall, 2002.

[2] T.Sun and Y. Neuvo, "Detail-preserving median based filters in image processing," Pattern Recognit. Lett., vol. 15, pp. 341-347, 1994.

[3] T.Chen and H. R. Wu, "Space variant median filters for the restoration of impulse noise corrupted images," IEEE Trans. Circuits Syst. II, Analog Digit. Signal Process., vol. 48, no. 8, pp. 784-789, Aug. 2001.

[4] "Adaptive impulse detection using center-weighted median filters," IEEE Signal Process. Lett., vol. 8, no. 1, pp. 1-3, Jan. 2001.

[5] P.S.Windyga, "Fast impulsive noise removal," IEEE Trans. Image Process., vol. 10, no. 1, pp. 173-179, Jan. 2001.

[6] N.Alajlan, M. Kamel, and E. Jernigan, "Detail preserving impulsive noise removal," Signal Process.: Image Commun., vol. 19, pp. 993-1003, 2004.

[7] E. Abreu, M. Lightstone, S. K. Mitra, and K. Arakawa, "A new efficient approach for the removal of impulse noise from highly corrupted images," IEEE Trans. Image Process., vol. 5, no. 6, pp. 1012-1025, Jun. 1996.

[8] G. Pok, J. C. Liu, and A. S. Nair, "Selective removal of impulse noise based on homogeneity level information," IEEE Trans. Image Process., vol. 12, no. 1, pp. 85-92, Jan. 2003.

[9] V. Crnojevic', V. ` Senk, and 'Z Trpovski, "Advanced impulse detection based on pixel-wise MAD," IEEE Signal Process. Lett., vol. 11, no. 7, pp. 589-592, Jul. 2004.

[10] I. Aizenberg, C. Butakoff, and D. Paliy, "Impulsive noise removal using threshold Boolean filtering based on the impulse detecting functions," IEEE Signal Process. Lett., vol. 12, no. 1, pp. 63-66, Jan. 2005.
[11] E.Bes, dok and M. E. Yüksel, "Impulsive noise suppression from images with Jarque-Bera test based median filter," J. Electron. Commun., vol. 59, pp. 105110, 2005.

[12] F.Russo, "Impulse noise cancellation in image data using a two-output nonlinear filter Measurement", vol.36, pp. 205-213, 2004.

[13] W.Luo, "A new efficient impulse detection algorithm for the removal of impulse noise," IEICE Trans. Fundam., vol. E88-A, no. 10, pp.

[14] M.N. Do and M. Vetterli, "Contourlets," in Beyond Wavelets, G. V. Welland, Ed. New York: Academic Press, 2003.

[15] "The contourlet transform: an efficient directional multiresolution image representation," IEEE Trans. Image Proc., appear,http://www.ifp.uiuc.edu/ $/$ minhdo/publications.

[16] Bo Xiong and Zhouping Yin, "A Universal Denoising Framework With a New Impulse Detector and Nonlocal Means", IEEE Trans. Image Process, VOL. 21, NO. 4, APRIL 2012.

[17] H.Hwang and R.A. Haddad, "Adaptive median filters: Newalgorithms and results," IEEE Trans. Image Process., vol. 4, no.4, pp. 499-502, Apr. 1995.

[18]Aditya Mavlankar, David Chen, Sameh Zakhary, Markus Flierl, and Bernd Girod "Noise Processing For Simple Laplacian Pyramid Synthesis Based on Dual Frame Reconstruction" Information System Laboratory Department of Electrical Engineering, Stanford UniversityStanford.

[19] Dr. G. Suganthi, M. Mohana Dhas ," High Density Saltand-Pepper Noise Reduction by combining NAFSM Filter and FCM" International Journal of engineering technology and computer applications Vol.1 No.2. 